\title{
Autonomous Orbit Determination for a Hybrid Constellation
}

\author{
Muzi Li $\left(\mathbb{D},{ }^{1}\right.$ Bo Xu $\left(\mathbb{D},{ }^{2}\right.$ and Jun Sun $^{3}$ \\ ${ }^{1}$ School of Astronomy and Space Science, Nanjing University, Nanjing 210023, China \\ ${ }^{2}$ School of Aeronautics and Astronautics, Sun Yat-Sen University, Guangzhou 510006, China \\ ${ }^{3}$ Shanghai Aerospace Control Technology Institute, Shanghai 201199, China \\ Correspondence should be addressed to Bo Xu; xubo@nju.edu.cn
}

Received 4 March 2018; Revised 6 August 2018; Accepted 13 August 2018; Published 26 September 2018

Academic Editor: Jose Carlos Páscoa

Copyright (c) 2018 Muzi Li et al. This is an open access article distributed under the Creative Commons Attribution License, which permits unrestricted use, distribution, and reproduction in any medium, provided the original work is properly cited.

\begin{abstract}
A new orbit determination scheme targeting communication and remote sensing satellites in a hybrid constellation is investigated in this paper. We first design one such hybrid constellation with a two-layer configuration (LEO/MEO) by optimizing coverage and revisit cycle. The main idea of the scheme is to use a combination of imagery, altimeter data, and inter-satellite range data as measurements and determine orbits of the satellites in the hybrid constellation with the help of the extended Kalman filter (EKF). The performance of the new scheme is analyzed with Monte Carlo simulations. We first focus on an individual remote sensing satellite and compared the performance of orbit determination using only imagery with its counterpart using both imagery and altimeter measurements. Results show that the performance improves when imagery is used with altimeter data pointing to geometer calibration sites but declines when used with ocean altimeter data. We then expand the investigation to the whole constellation. When inter-satellite range data is added, orbits of all the satellites in the hybrid constellation can be autonomously determined. We find that the combination of inter-satellite range data with remote sensing observations lead to a further improvement in orbit determination precision for LEO satellites. Our results also show that the performance of the scheme would be affected when remote sensing observations on certain satellites are absent.
\end{abstract}

\section{Introduction}

A hybrid constellation refers to a group of satellites at different orbital regimes working in concert. A widely known example is the BEIDOU navigation satellite constellation of China. The hybrid constellation concept commonly appears in studies on navigation constellation design and optimization $[1,2]$. It has also been introduced to the fields of satellite communication and remote sensing [3,4], and Fahnestock and Erwin [5] presented a kind of hybrid constellation to meet space situation awareness requirements. However, few research tries to design a hybrid constellation as a multifunction constellation.

At present, orbit determination for satellites depends heavily on the earth stations and navigation constellations (for example, GPS constellation). In order to reduce maintenance cost of these systems and enhance survivability of satellites in cases of emergencies, autonomous orbit determination methods which depend on instruments on board were proposed. Some of these works focus on autonomous orbit determination based on either optical imagery or altimeter data or inter-satellite range data. White et al. [6] used lineof-sight (LOS) measurements of stars and landmarks to estimate the attitude and orbit of satellites. Straub and Christian [7] used observations of coastlines on the Earth's surface as inputs to autonomously determine the orbits of earthobserving satellites with different orbit inclinations and altitudes. Li, Xu and Zhang [8] proposed a scheme using images of ground objects and analyzed the influence of image resolution, pointing accuracy and lighting constraints on the orbit determination performance. Following that study, Li and $\mathrm{Xu}$ [9] presented an orbit and attitude determination (OAD) scheme using images of regular-shaped ground landmarks to overcome the disadvantages using ground point features. As another high-precision measurement, the altimeter can provide highly accurate altitude information which helps to effectively improve the orbit determination accuracy. Born et al. [10] determined the orbit of the N-ROSS satellite 
using the altimetric crossing arc residuals between the TOPEX and N-ROSS orbits and demonstrated that submeter radial accuracy can be attained. Lemoine et al. [11] showed that altimeter crossover data can significantly modify the gravity field and improve the radial orbit accuracy of POD to $4-5 \mathrm{~cm}$ for the GEOSAT Follow-On spacecraft when used in combination with SLR data. For satellites in constellations, intersatellite links can be established and pseudo-range observations of these links can be used for orbit determination. Markley and Naval [12] investigated orbit determination performance using landmarks and intersatellite data. Psiaki [13] proposed an autonomous orbit determination system based on the relative position measurement of a pair of satellites and analyzed the observability and orbit estimation accuracy of the system. Li et al. [14] verified the possibility of reducing the errors resulting from constellation rotation by using cameras to obtain the direction between satellites. Kai et al. [15] evaluated the performance of a navigation scheme which uses relative bearing measurements from navigation star sensors combined with relative range measurements from intersatellite links. Besides, Kai et al. [16] introduced a scheme using the time difference of arrival (TDOA) measurements to X-ray pulsars and inter-satellite range measurements to determine the absolute position of satellites. Wang and Cui [17] also achieved autonomous navigation using the X-ray pulsars and inter-satellite range measurements for Mars obiters.

In this paper, unlike previous studies on hybrid constellations which focus on satisfying a specific requirement of communication, navigation, or remote sensing, a hybrid constellation containing two layers (MEO/LEO) was proposed to meet both the requirements of satellite communication and remote sensing. The LEO layer satellites with optical cameras and altimeters onboard are designed for Earth observation, and the MEO layer is designed in combination with the LEO layer to be a communication constellation. For the hybrid constellation, a new orbit determination scheme is proposed. Without other observation data external to the constellation, only optical imagery and altimeter data can be used as high-precision observations for autonomous orbit determination of LEO satellites. Two usage patterns are considered for the altimetry data. One is the ocean altimeter data generated with nadir-pointing altimeters. The other is the range data generated with altimeters pointing to the geometer calibration sites which can be captured and recognized by the camera systems. When inter-satellite range data is considered, orbits of MEO layer satellites can also be determined, which in turn has an effect on the LEO layer satellites. As a result, autonomous orbit determination of the constellation containing communication satellites and remote sensing satellites can be achieved.

Under such a constellation, the performance of autonomous orbit determination using optical imagery, altimeter data, and inter-satellite range data is evaluated. For altimeter data-based orbit determination, the influence of different usage patterns on orbit accuracy is compared. For orbit determination using all three observation data, the performance is also assessed in the circumstance when certain remote sensing observations are absent.
To this end, the remainder of this paper is organized as follows: Section 2 introduces the optimal hybrid constellation. In Section 3, a detailed description of the orbit determination algorithm is given, including the dynamic model, the measurement model, and the filter model. For different observation data, orbit determination simulations and performance analysis are shown in Section 4. Finally, some brief conclusions and discussions are provided in Section 5 .

\section{Hybrid Constellation Design}

In this section, a hybrid constellation consisting of MEO/LEO two layer satellites is proposed. The LEO layer is designed to implement an Earth optical observation mission. Besides, the LEO layer cooperating with the MEO layer can provide continuous regional communication coverage. Considering orbit characteristics of the hybrid constellation and related constraints, an efficient design procedure is presented below.

2.1. LEO Layer. To ensure the accuracy of obtained data, satellites performing earth observation missions are mostly placed on LEO. In the paper, the LEO layer is designed as a remote sensing constellation satisfying coverage and revisit cycle requirements. Given that the satellites placed on sunsynchronous orbit pass over a given sub-satellite point at a fixed local solar time, the sun-synchronous orbit is appropriate for earth observation satellites and satisfies the nondimensional form [18]:

$$
\dot{\Omega}=-\frac{3}{2} J_{2} \frac{n \cos i}{p^{2}}=n_{s}
$$

where $\Omega$ is the right ascension of ascending node, $J_{2}$ is the second zonal harmonic of the gravitational field, $n$ is the mean motion of the satellite, $p$ is the semi-latus rectum of the orbit, and $n_{s}$ is the mean angular velocity of the Earth orbiting the Sun. On the basis of the sun-synchronous orbit, a further assumption is made that the orbits meet the conditions of a repeat circular orbit:

$$
\begin{aligned}
N_{p} T_{\Omega} & =N_{d} T_{E}, \\
T_{E} & =\frac{2 \pi}{n_{e}-\dot{\Omega}}, \\
T_{\Omega} & =\frac{2 \pi}{n}\left(1+\frac{3}{2} J_{2} \frac{1-4 \cos ^{2} i}{p^{2}}\right),
\end{aligned}
$$

where $N_{p}$ represents the number of revolutions in one repetition, $N_{d}$ is the number of days to repeat (revisit cycle), $n_{e}$ is the inertial rotational velocity of the Earth, $T_{\Omega}$ is the nodal period of the orbit, and $T_{E}$ is the rotational period of the Earth. Substituting (2), (3), and (4) into (1), a nonlinear mathematical equation with a single variable "the semimajor axis $a$ " can be written as

$$
A_{1} a^{7}+A_{2} a^{2}+A_{3} a^{0.5}+A_{4}=0
$$


where the expressions of the coefficients are as follows:

$$
\left\{\begin{array}{l}
A_{1}=\frac{8\left(n-n_{s}\right) n_{s}^{2}}{3 J_{2}}, \\
A_{2}=n_{e}-n_{s} \\
A_{3}=\frac{-N_{d}}{N_{p}}, \\
A_{4}=\frac{3 J_{2}\left(n_{e}-n_{s}\right)}{2} .
\end{array}\right.
$$

For (5), there are some constraint conditions: (1) In order to ensure good revisit performance and less computation burden, the revisit cycle is set no more than 10 days; (2) For a remote sensing satellite bus orbiting in LEO, the orbit altitude normally ranges between $500 \mathrm{~km}$ and $1000 \mathrm{~km}$. Therefore, the orbit revolution per day is limited to more than 14 and less than 15; (3) Satellites with a total number of $N_{s}$ are uniformly distributed on the same orbit. To make sure that intersatellite links can be established between adjacent satellites, the number of satellites is not less than the minimum number which keeps adjacent satellites visible to each other, meanwhile the number of satellites is required to be no larger than 2 times of this minimum number in order to limit the constellation size; (4) In the nadir viewing case, the single-plane constellation can provide complete coverage of the Earth (except polar regions). The field of view (FOV) of every satellite is set as $2.06^{\circ}$ which equals the FOV of satellites in the high-resolution satellite constellation "DMC-3G" [19]. The corresponding mathematical expressions (the derivation is provided in Appendix A) are shown as

$$
\left\{\begin{array}{l}
N_{d} \leq 10, \\
14 \leq \frac{N_{p}}{N_{d}} \leq 15, \\
\frac{\pi}{\arccos \left(r_{e} / a\right)} \leq N_{s} \leq \frac{2 \pi}{\arccos \left(r_{e} / a\right)}, \\
\frac{2\left(a-r_{e}\right) \tan (\mathrm{FOV} / 2)}{|\sin i|}>2 \pi r_{e} \frac{N_{d}}{\operatorname{LCM}\left(N_{d}, N_{s}\right) N_{p}},
\end{array}\right.
$$

where $r_{e}$ is the equatorial radius. Under these constraints and repeat sun-synchronous orbit equation, the number of feasible solutions is finite. By going through all the possible combinations of $N_{d}$ and $N_{p}$, all the feasible solutions can be generated. Then the solution with the minimum sum of $N_{d}$ and $N_{s}$ is identified as the optimal solution (shown in Table 1). Among the two solutions, the first solution with the minimal orbit altitude is the optimal choice.

In order to guarantee good illumination, the descending node local time is set around 10:30 am or 1:30 pm for most LEO Earth observation satellites. Here the single plane determined by (5) is set with a descending node local time of 10:30 am. For further decreasing the revisit cycle, an orbit plane with a descending node local time of 1:30 pm is added under the Flower Constellation concept. The Flower
TABLE 1: The optimal results of parameters for a single plane of the LEO layer.

\begin{tabular}{lcccc}
\hline Altitude $(\mathrm{km})$ & Inclination $\left(^{\circ}\right)$ & $\boldsymbol{N}_{p}$ & $\boldsymbol{N}_{d}$ & $\boldsymbol{N}_{\boldsymbol{s}}$ \\
\hline 811.17 & 98.66 & 128 & 9 & 11 \\
848.91 & 98.82 & 127 & 9 & 11 \\
\hline
\end{tabular}

Constellation has the property that all satellites share identical repeat ground tracks [20], which helps to reduce revisit cycle. For satellites in a Flower Constellation, the difference of the right ascension of ascending node and the difference of mean anomaly $\Delta M$ satisfies

$$
\left\{\begin{array}{l}
\Delta \Omega=-2 \pi \frac{F_{n}}{F_{d}} \\
\Delta M=2 \pi \frac{F_{n}}{F_{d}}\left(\frac{n+\dot{M}_{0}}{n_{e}+\dot{\Omega}}\right)
\end{array}\right.
$$

in which $F_{n}$ and $F_{d}$ are the phasing parameters and $\dot{M}_{0}$ is the rate of change in the mean anomaly due to perturbations. $\Delta \Omega$ is $45^{\circ}$ which can be calculated by subtracting between the descending node local time of two orbit planes, and then the ratio $F_{n} / F_{d}$ and $\Delta M$ can be obtained. A total of $N_{s}$ satellites are uniformly distributed in the first plane, so the same number of $N_{s}$ satellites are placed in the second plane to repeat the corresponding satellite ground track. From (8), it can be inferred that there exist $N_{d}$ positions in one plane which share the same ground track [21]; the mean anomaly separation between adjacent positions is $2 \pi / N_{d}$. The minimum and the maximum revisit cycles for these $N_{d}$ positions are shown in Table 2. In order to minimize the maximum revisit cycle, $\Delta M=213.89^{\circ}$ is chosen. Finally, the maximum revisit cycle of LEO layer satellites is reduced to 117 hours.

2.2. MEO Layer. The MEO layer is constructed to work in conjunction with the LEO layer for continuous regional communication. As communication satellites, GEO satellites do well in covering low latitudes; however, their long roundtrip time make it difficult for GEO satellites to provide quality-guaranteed service for real-time need. What is more, the position resource on GEO satellites is scant. The MEO and LEO satellites greatly overcome the disadvantages of GEO satellites. When compared with MEO satellites, LEO satellites have a better quality of timeliness, while more LEO satellites would be needed to achieve continuous global or regional coverage. Therefore, a hybrid constellation including the MEO layer and LEO layer would be suitable to be used for regional communication.

For the design of the MEO layer, the Walker Delta constellation concept [22] is adopted. This constellation can provide an excellent coverage and is defined by four parameters $(i / T / W / F)$. The first parameter $i$ is the inclination of the satellite orbit. $T$ is the total number of satellites. $W$ is the number of orbital planes, and $F(0 \leq F \leq W-1)$ is the phase factor which denotes the relative phases of the satellites in any two adjacent planes. In the paper, the MEO layer is 
TABLE 2: The minimum and the maximum revisit cycles.

\begin{tabular}{cccc}
\hline & $\begin{array}{c}\Delta M \\
\text { (degree) }\end{array}$ & $\begin{array}{c}\text { Minimum revisit cycle } \\
\text { (hours) }\end{array}$ & $\begin{array}{c}\text { Maximum revisit cycle } \\
\text { (hours) }\end{array}$ \\
\hline 1 & 82.98 & 3 & 213 \\
2 & 115.71 & 27 & 189 \\
3 & 148.43 & 51 & 165 \\
4 & 181.16 & 75 & 141 \\
5 & 213.89 & 99 & 117 \\
6 & 246.61 & 93 & 123 \\
7 & 279.34 & 69 & 147 \\
8 & 312.07 & 45 & 171 \\
9 & 344.80 & 21 & 195 \\
\hline
\end{tabular}

required to cooperate with the LEO layer to realize continuous coverage of the latitude zone including the China mainland $\left(3.86^{\circ} \mathrm{N} \sim 53.55^{\circ} \mathrm{N}\right)$. Parameters $T, W$, and $F$ can be empirically chosen as $T=8, W=2$, and $F=1$. The orbit altitude and inclination are important factors affecting the coverage rate and minimum elevation angle in this design. The higher the altitude is, the better the visibility of the communication satellite is. Here the orbit altitude can be set according to the 8-hour circular periodic orbit. As for the inclination, Figure 1 shows the relation between the minimum elevation angle and the inclination under the constraint of continuous coverage. Given the requirement of a minimum elevation angle higher than 20 degrees, choice of the inclination is limited between 36 and 42 . Figure 2 shows the variation of coverage rate with inclination. When inclination is set around 40, coverage rate reaches a peak. Here the inclination is set at 40 , which guarantees a larger minimum elevation angle and a continuous coverage at the same time. The overall design configuration of the hybrid constellation is summarized in Table 3.

\section{Orbit Determination Scheme}

This section presents the orbit determination scheme for the constellation designed in Section 2, including a description of the dynamic model, the measurement model, and the filter model to estimate orbit states.

3.1. Dynamic Model. For the constellation, the state vector is represented as $\mathbf{x}=\left[\mathbf{x}_{1}^{T} \mathbf{x}_{2}^{T} \cdots \mathbf{x}_{1}^{T}\right]$ where $\mathbf{x}_{i}=\left[\mathbf{r}_{i} \dot{\mathbf{r}}_{i}\right]^{T}, \mathbf{r}_{i}=\left[\mathbf{r}_{x, i}\right.$ $\left.\mathbf{r}_{y, i} \mathbf{r}_{z, i}\right]$, and $\dot{\mathbf{r}}_{i}=\left[\mathbf{v}_{x, i} \mathbf{v}_{y, i} \mathbf{v}_{z, i}\right]$ are position and velocity vectors of the satellites in J2000 Earth-centered inertial coordinate system. The subscripts $i$ and $l$ indicate the $i$-th satellite and the total number of satellites, respectively. The equation of motion for a satellite is described as

$$
\dot{\mathbf{x}}=f(\mathbf{x}, t),
$$

where

$$
f(\mathbf{x}, t)=\left[\begin{array}{c}
\dot{\mathbf{r}} \\
\frac{-\mu}{\|\mathbf{r}\|^{3}} \mathbf{r}+\mathbf{F}_{\varepsilon}
\end{array}\right],
$$

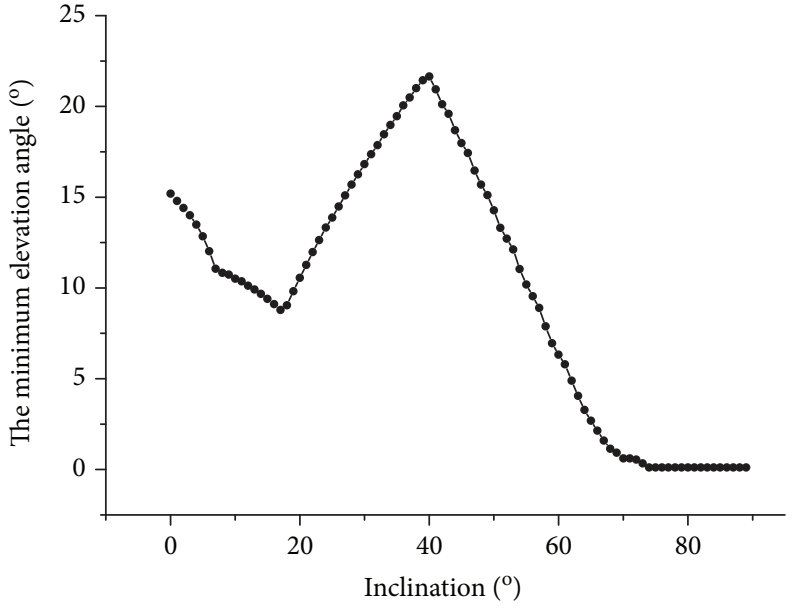

Figure 1: The relation between the minimum elevation angle and the inclination.

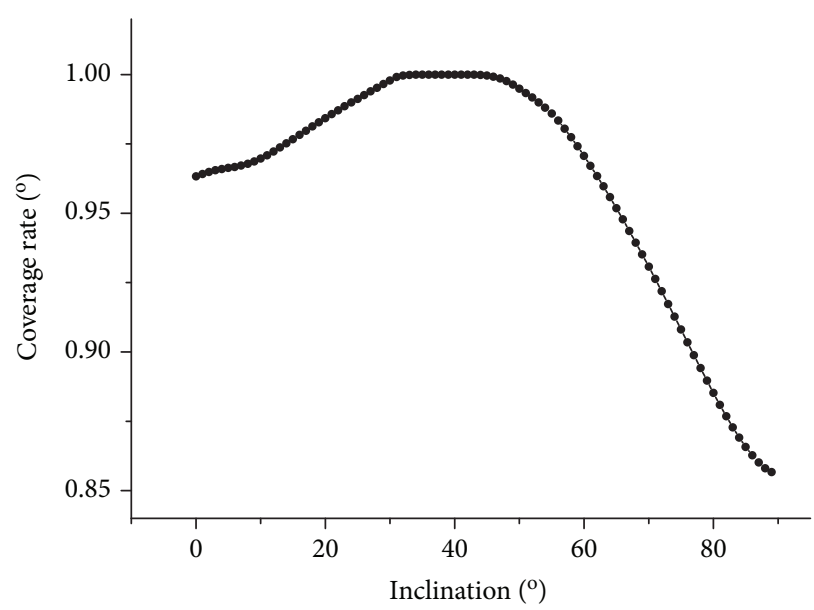

FIGURE 2: Variation of the coverage rate with the inclination.

TABLE 3: Initial orbit element of the hybrid constellation.

\begin{tabular}{lcc}
\hline & LEO layer & MEO layer \\
\hline Satellite number & 22 & 8 \\
Plane number & 2 & 2 \\
Semi-major axis $(\mathrm{km})$ & 7189.31 & 20268.43 \\
$\begin{array}{l}\text { Eccentricity } \\
\text { Inclination }\left(^{\circ}\right)\end{array}$ & 0 & 0 \\
Right ascending node $\left(^{\circ}\right)$ & 98.66 & 40 \\
$\begin{array}{l}\text { Argument of perigee plus mean } \\
\text { anomaly for the first satellite of } \\
\text { every plane }\left({ }^{\circ}\right)\end{array}$ & $0 / 213.90$ & $0 / 180$ \\
\hline
\end{tabular}

$\mu$ is the earth gravitational constant, and $F_{\varepsilon}$ represents perturbation accelerations. The sources of perturbations include the nonspherical gravitation of the Earth (the WGS84 gravity model), the atmospheric drag (1976 standard atmosphere model), third-body perturbations (DE405 numerical 
planetary ephemeris), solar radiation pressure (spherical model), Earth radiation pressure, the tide effects, and the effect of general relativity.

3.2. Measurement Model. In this paper, optical imagery, altimeter data, and inter-satellite range data are used to provide the orientation, altitude, and relative distance information, respectively, for the constellation satellites.

For remote sensing satellites with an optical camera, images of known ground features can be captured. By the process of image recognition and matching, the focal plane coordinates of a ground feature are obtained, which imply the orientation information of the cameras to ground features. The focal plane coordinates $(u, v)$ satisfy

$$
\left[\begin{array}{l}
u \\
f \\
v \\
\bar{f} \\
1
\end{array}\right]=\left(\mathbf{T}_{A} \mathbf{T}_{I}\right)^{-1}\left[\mathbf{r}-\mathbf{r}_{g}\right]
$$

$\mathbf{T}_{I}$ and $\mathbf{T}_{A}$ are the camera installation matrix and the rotation matrix from the satellite body coordinate frame to the J2000 initial coordinate frame, respectively. It is assumed that a good estimation of $\mathbf{T}_{A}$ can be available from a star tracker. $f$ is the focal length of the optical camera, and $\mathbf{r}_{g}$ is the ground feature position. Simplifying (11) yields

$$
\left[\begin{array}{l}
u \\
v
\end{array}\right]=\mathbf{g}(\mathbf{x})+\boldsymbol{\eta},
$$

where $\boldsymbol{\eta}$ denotes the measurement noise satisfying the zeromean Gaussian distribution

$$
\mathrm{E}\left(\boldsymbol{\eta} \boldsymbol{\eta}^{\mathrm{T}}\right)=\sigma_{i}^{2} \mathbf{I}_{2 \times 2}
$$

in which $\sigma_{i}$ is the standard deviation of the focal plane coordinate error and $\mathbf{I}_{2 \times 2}$ is a two-order identity matrix.

The laser altimeter measurement belongs to the twoway range measurement. The laser altimeter sends a laser beam and measures the round-trip time. The range can be described as

$$
\rho_{a}=\frac{c t_{a}}{2}
$$

in which $c$ is the speed of light, $t_{a}$ is the round-trip time, and $\rho_{a}$ is the altimeter measurement. By adjusting the orientation of the altimeter antenna boresight, the altimeter can be directed toward the nadir to obtain height information above the Earth's surface or toward interested regions to measure the range between each other.

When the altimeter is directed toward the nadir, the range measurement is described as

$$
\rho_{a}=\left|\mathbf{r}-\mathbf{r}_{u}\right|+\zeta=|\mathbf{r}|-r_{e}\left(1-f_{e} \sin ^{2} \theta\right)+\zeta,
$$

in which $\mathbf{r}_{u}$ is the position of the sub-satellite point, $f_{e}$ is the flattening of the Earth, and $\theta$ is the geocentric latitude. The range to interested regions measured by the altimeter is expressed as

$$
\rho_{a}=\left|\mathbf{r}-\mathbf{r}_{p}\right|+\zeta
$$

where $\mathbf{r}_{p}$ is the position of the interested point and $\zeta$ is the measurement noise which satisfies

$$
E\left(\zeta^{2}\right)=\sigma_{a}^{2}
$$

where $\sigma_{a}$ is the standard deviation of the altimeter measurement error.

For inter-satellite range measurement, the observed inter-satellite range $\rho_{s}$ can be represented by

$$
\rho_{s, i j}=\left|\mathbf{r}_{j}-\mathbf{r}_{i}\right|+\chi
$$

in which the subscripts $j$ and $i$ represent the $j$-th and $i$-th satellites, respectively, and $\chi$ presents the random measurement noise which satisfies

$$
E\left(\chi^{2}\right)=\sigma_{s}^{2}
$$

In (19), $\sigma_{s}$ is the standard deviation of the inter-satellite range error.

3.3. Filter Model. Taking into account that the satellite orbit dynamics and measurement model are nonlinear, an EKF is used to estimate the state of the system. Equation (9) is used as the state equation, and (12), (15), and (18) represent the observation equations for different observations. The EKF works by a two-step cycle: a time update step and a measurement update step [23].

The covariance $\mathbf{P}$ propagation from time $k-1$ to the next observation epoch $k$ is processed with the "time update" equation:

$$
\overline{\mathbf{P}}_{k}=\boldsymbol{\Phi}\left(t, t_{k}\right) \mathbf{P}_{k-1} \boldsymbol{\Phi}^{T}\left(t, t_{k}\right),
$$

where $\boldsymbol{\Phi}\left(t, t_{k}\right)$ is the state transformation matrix. The state $\mathbf{x}$ and covariance $\mathbf{P}$ are updated with the observation using the "measurement update" equation:

$$
\begin{aligned}
\mathbf{K}_{k} & =\overline{\mathbf{P}}_{k} \mathbf{H}_{k}^{T}\left(\mathbf{H}_{k} \overline{\mathbf{P}}_{k} \mathbf{H}_{k}^{T}+\mathbf{R}_{k}\right)^{-1}, \\
\widehat{\mathbf{x}}_{k} & =\mathbf{K}_{k} \mathbf{y}_{k}, \\
\mathbf{x}_{k} & =\mathbf{x}_{k}+\widehat{\mathbf{x}}_{k}, \\
\mathbf{P}_{k} & =\left(\mathbf{I}-\mathbf{K}_{k} \mathbf{H}_{k}\right) \overline{\mathbf{P}}_{k},
\end{aligned}
$$

where $\mathbf{H}_{k}$ is the measurement sensitivity matrix which is the partial derivative of measurements with respect to the state vector, $\mathbf{K}_{k}$ is the gain matrix, $\widehat{\mathbf{x}}_{k}$ is the state deviation, and $\mathbf{y}_{k}$ is the observation residual. The measurement sensitivity matrixes can be derived according to the observation 
equations. For different measurement models, the measurement sensitivity matrixes can be expressed as follows:

For image measurement (the detailed formula is in Appendix B),

$$
\mathbf{H}_{\mathbf{k}}^{\mathbf{i}}=\frac{\partial(u, v)}{\partial \mathbf{r}}
$$

For the altimeter measurement to interested regions,

$$
\mathbf{H}_{\mathbf{k}}^{\mathbf{a}}=\left[\frac{\left(\mathbf{r}-\mathbf{r}_{p}\right)^{T}}{\left|\mathbf{r}-\mathbf{r}_{p}\right|} 0_{1 \times 3}\right]
$$

For altimeter measurement toward the nadir,

$$
\begin{aligned}
\mathbf{H}_{\mathbf{k}}^{\mathbf{a}}= & {\left[\frac{r_{x}}{|r|}-\frac{2 r_{e} f_{e} r_{x} r_{z}^{2}}{|r|^{4}} \frac{r_{y}}{|r|}-\frac{2 r_{e} f_{e} r_{y} r_{z}^{2}}{|r|} \frac{r_{z}}{|r|}\right.} \\
& \left.-\frac{2 r_{e} f_{e} r_{z}\left(r_{x}^{2}+r_{y}^{2}\right)}{|r|} 0_{1 \times 3}\right] .
\end{aligned}
$$

For inter-satellite range measurement (the homologous part of one satellite in the link),

$$
\mathbf{H}_{\mathbf{k}}^{\mathbf{s}}=\left[\begin{array}{ll}
\frac{\left(\mathbf{r}-\mathbf{r}_{i}\right)^{T}}{\left|\mathbf{r}-\mathbf{r}_{i}\right|} & 0_{1 \times 3}
\end{array}\right]
$$

Once a new observation is obtained, the state is updated. The updated state is propagated forward to the next observation time, and the filter continues until all measurements are processed.

\section{Simulation Results}

In the section, orbit determination performance of simulations using different strategies for the hybrid constellation are evaluated and compared.

4.1. Initial Condition. The initialization was set at the epoch (1 July 2009, 10:30:00), and the initial orbit elements are shown in Table 3. Nominal orbit data of the hybrid constellation was generated considering all perturbations referred to in Section 3.1. The dynamic model used in orbit determination includes (1) the two-body gravitation, (2) the nonspherical gravitation (the WGS84 $20 \times 20$ gravity), (3) the atmospheric perturbation (1976 standard atmosphere model), (4) the gravitation of the Moon and Sun (DE405 numerical planetary ephemeris), (5) the solar radiation pressure (spherical model), and (6) the perturbation of rigid tides.

For MEO layer satellites, only inter-satellite range data can be used to determine orbit, while for LEO layer satellites, the inter-satellite range data, optical imagery, and altimeter data can all be used to determine orbit. The visibility limitation was taken into consideration while generating inter-satellite range data. For optical imagery, when the sub-satellite region is at night or covered by clouds, no effective images can be

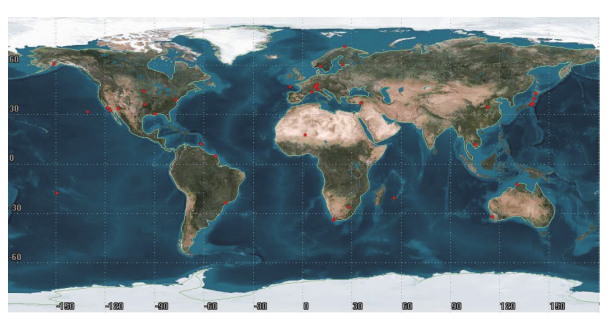

FIgure 3: The distribution of the geometric calibration sites on the Earth's surface.

achieved by satellites. The probability that the imaged region is covered by clouds is set as $50 \%$. Measurement errors from different sources were simulated by Gaussian zero-mean random error for three measurement data. The uncertainty of inter-satellite range was set as $1 \mathrm{~m}(1 \sigma)$. For optical imagery, the measurement error of focal plane coordinates mainly results from three sources: the image resolution was set as $1 \mathrm{~m}(1 \sigma)$, the pointing accuracy was chosen as $0.001^{\circ}$, and the coastline and rivers were used as ground features with a $10 \mathrm{~m}(1 \sigma)$ position error [8]. For the altimeter data, although the altimeter precision can reach the level of centimeters, the height measuring accuracy used in orbit determination is also influenced by several other factors. For example, the difference between the actual Earth ground and the geodetic reference ellipsoid is the main factor leading to the height measurement error [23]. Besides, due to beam divergence, the altimeter range measurement is the average of the ranges from the altimeter to the footprint of each laser beam on the Earth surface. The effect of this averaging could be decreased when only ocean altimeter data is used, as ocean topography is flatter than that of land. Considering these facts, a $10 \mathrm{~m}$ $(1 \sigma)$ error was added to ocean altimeter data with the help of the precise Earth shape and tide model. Unlike the traditional usage pattern of the altimeter, a laser altimeter can be used to measure the range between satellites and some interested regions when used in combination with optical cameras. This is achieved by making the laser altimeter point to the ground features that can be captured and recognized by optical cameras. The geometer calibration sites applied in mission SPOT6 and provided by ESA [24] were used as the ground targets in this paper. The site distribution is shown in Figure 3. The small number of ground sites would make it hard to capture them. Therefore, it is assumed that the satellites can swing (the swing angle is no more than $30^{\circ}$ ) to point to these sites, when approaching them. In such a situation, the altimeter measurement error was set as $1 \mathrm{~m}(1 \sigma)$.

In these scenarios, all measurements were obtained with an interval of $1 \mathrm{~min}$. The inter-satellite range data and altimeter data are generated by the nominal orbit data and Reference Earth Model (WGS84). Due to the use of synthetic data instead of real satellite imagery, the image processing stage is not considered in this article. Thus, related latency is not involved in the simulations. The observed coordinates of ground features on the focal plane are generated through geometric relationships between the positions of ground features and the actual orbit, under the nadir imaging model. 

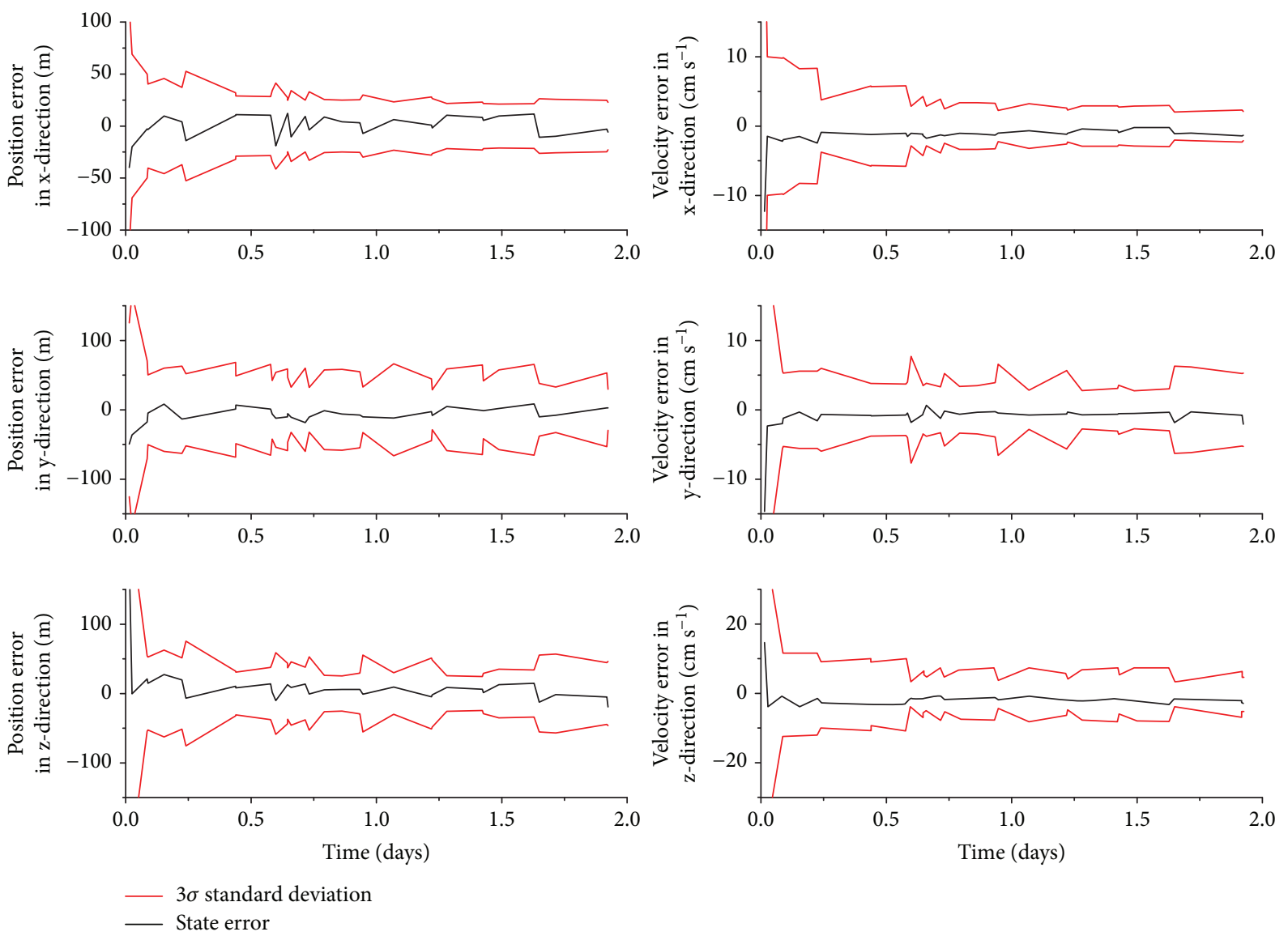

FIgURE 4: Time history of position and velocity errors of a LEO layer satellite in the J2000 coordinate frame for the case using only optical imagery.

For the satellite orbit, the initial position error was $100 \mathrm{~m}$ and the initial velocity error was set as $0.1 \mathrm{~cm} / \mathrm{s}$.

4.2. Results and Analysis. In this part, results of case simulations using different orbit determination strategies are analyzed in detail.

The comparison was first conducted between the case using only optical imagery and the case using either altimeter data. As the orbit determination results are similar for all LEO layer satellites under these two scenarios, only results of the first satellite in the LEO layer are shown. Figure 4 shows the time history of orbit error only using optical imagery, and the orbit determination results using optical imagery and altimeter data as inputs are presented in Figures 5 and 6. In these figures, the black line in each plot denotes the actual orbit error, and the red line indicates the $3 \sigma$ standard deviation of the estimated error. It can been seen that for all cases, the actual errors are well-bounded by the 3-sigma standard deviations and drop significantly to a steady state in less than 0.2 days. Because the measurement and dynamic model are nonlinear, a Monte Carlo test of 100 runs was performed to further compare orbit determination accuracy, and the average RMS errors are given in Table 4. In the case using ocean altimeter data and optical imagery, the results get worse compared with the case using only optical imagery. The average
RMS errors increase by a factor of 2.30 for position error and 1.90 for velocity error. In general, the EKF should be able to filter out measurements characterized by higher error; thus, the orbit determination accuracy would not decrease when more measurements are added to an EKF. However, the result draws the opposite conclusion in this simulation. This is mainly due to the small amount of valid images. Although the optical imagery is set to be captured at a high frequency with an interval of $1 \mathrm{~min}$, only a small fraction of the images that contain a ground feature can be used as valid observation. Factors including small FOV, the discontinued distribution of the ground feature, and existence of light constraints would lead to the absence of a ground feature in some images and result in a lower measurement frequency of valid imagery compared to that of the ocean altimeter data. What is more, the ocean altimeter data only provides radial measurement and is not precise enough. Therefore, the orbit precision decreases when the ocean altimeter data is added. On the contrary, in the case using altimeter data pointing to the geometer calibration sites and optical imagery, a better performance than the case using only optical imagery is achieved, with a $41.35 \%$ enhancement in position accuracy and $45.14 \%$ enhancement in velocity accuracy.

In the case using optical imagery, altimeter data, and inter-satellite range data, the orbits of both LEO layer 

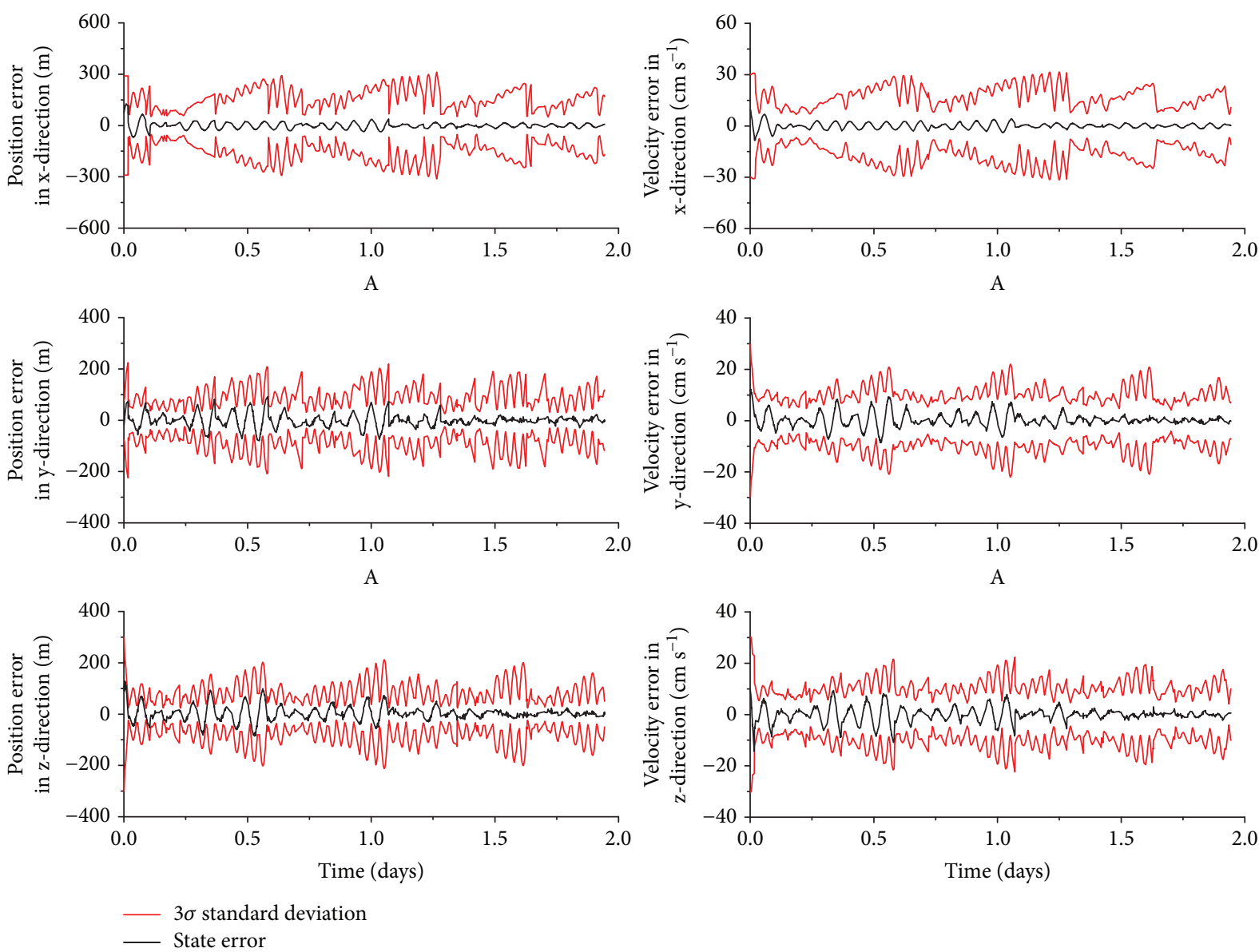

FIGURE 5: Time history of position and velocity errors of a LEO layer satellite in the J2000 coordinate frame for the case using optical imagery and ocean altimeter data.

satellites and MEO layer satellites can be determined simultaneously with the help of inter-satellite range data. The time history of orbit errors for a LEO satellite and a MEO satellite is show in Figures 7 and 8. It is obvious that the position error and velocity error further decrease compared with the cases discussed before. This conclusion is also confirmed by results from the Monte Carlo runs in Table 4. The average RMS errors of position and velocity drop to $47.24 \%$ and $42.70 \%$, respectively, compared to those in the case using optical imagery and altimeter data. The improvement in orbit accuracy can be explained as follows: to the multiplane, multilayer structure of the hybrid constellation, a good geometry configuration for relative range measurement is established. Under this configuration, the high-precision inter-satellite range data can effectively improve orbit determination accuracy in all directions.

In the above-mentioned case, all three data including optical imagery, altimeter data, and inter-satellite range data are used for orbit determination. However, in actual missions, the remote sensing instruments may have malfunction or get disturbed, and there are circumstances that LEO layer satellites are not loaded with both optical cameras and altimeters due to their mission requirements. These situations would make it impossible for certain LEO layer satellites to generate effective remote sensing observations. Thus, another two scenarios where there is a lack of remote sensing observation for certain LEO layer satellites are discussed. One (scenario 1) assumes that satellites on the first plane of the LEO layer fail to obtain altimeter measurement while other LEO satellites can get all three measurement data. The other (scenario 2) assumes that the satellites on the first plane of the LEO layer fail to obtain both imagery and altimeter data while other satellites can get all three measurement data. The same Monte Carlo runs were applied to these two scenarios, and simulated results are compared with the ideal case mentioned above in which all satellites in the LEO layer can obtain three measurement data. As is shown in Table 5, for the same scenario, there is no significant difference on the orbit accuracy between LEO satellites with and without a loss of remote sensing observation. Taking scenario 1 as an example, the change of position error (from 9.03 to 9.05) and velocity error (from 0.94 to 0.94 ) is almost negligible. However, between different scenarios, there exists significant difference on the average RMS of position and velocity errors. As long as there is absence of remote sensing observations, the orbit determination precisions for all satellites in the hybrid constellation decline significantly compared to the ideal scenario. 

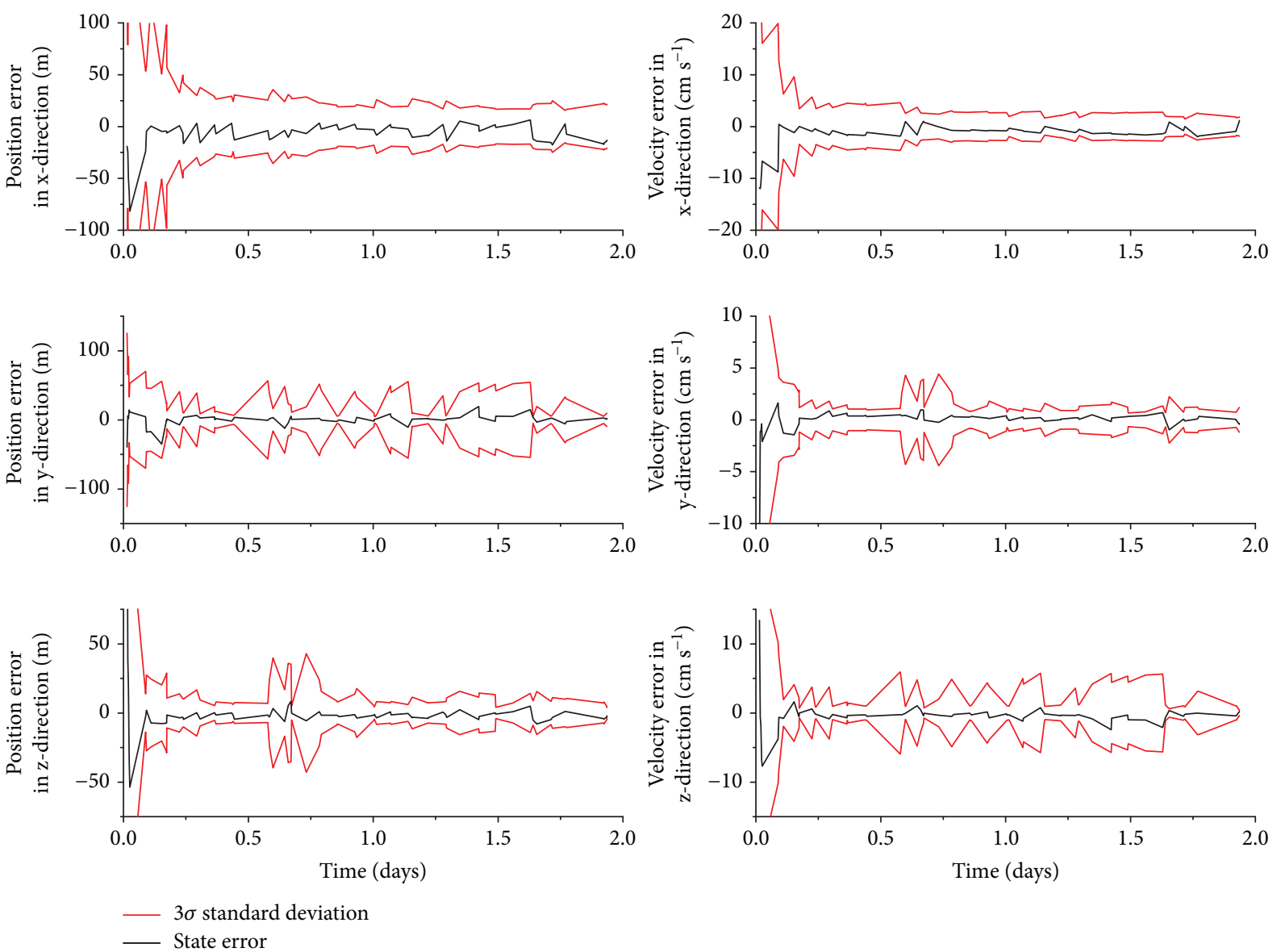

FIGURE 6: Time history of position and velocity errors of a LEO layer satellite in the J2000 coordinate frame for the case using optical imagery and range data to the geometric calibration sites.

TABLE 4: Average RMS errors of 100 Monte Carlo runs for different orbit determination scenarios.

\begin{tabular}{|c|c|c|c|c|}
\hline & \multicolumn{4}{|c|}{ RMS error } \\
\hline & \multicolumn{2}{|c|}{ LEO layer satellite } & \multicolumn{2}{|c|}{ MEO layer satellite } \\
\hline & Position (m) & Velocity $(\mathrm{cm} / \mathrm{s})$ & Position (m) & Velocity $(\mathrm{cm} / \mathrm{s})$ \\
\hline Image & 14.22 & 1.75 & - & - \\
\hline Image + altimeter (ocean) & 32.77 & 3.32 & - & - \\
\hline Image + altimeter (the geometer calibration site) & 8.34 & 0.96 & - & - \\
\hline Image + altimeter (the geometer calibration site) + inter-satellite range & 3.94 & 0.41 & 9.06 & 0.38 \\
\hline
\end{tabular}

\section{Conclusions}

In this paper, we proposed an autonomous orbit determination scheme for hybrid constellations by using a combination of three measurement data including optical imagery, altimeter data, and inter-satellite range data. By applying the scheme to a constructed hybrid constellation, the performance of the scheme is then investigated. The hybrid constellation consists of satellites in two LEO/MEO layers. The LEO layer is designed for Earth observation, which is capable of providing complete coverage of the Earth (except polar regions) and has a maximum revisit cycle of 117 hours. The
MEO layer is designed in conjunction with the LEO layer for communication and is capable of providing continuous coverage of latitude zone including China mainland.

With the help of measurement from onboard optical cameras and altimeters, orbits of individual LEO satellites can be autonomously determined. Comparison results show that a better performance is achieved when optical imagery is used in combination with altimeter data pointing to the geometer calibration sites than with ocean altimeter data. For MEO satellites, orbits can be determined autonomously by considering inter-satellite range data in combination with remote sensing observations, which in turn lead to a further 

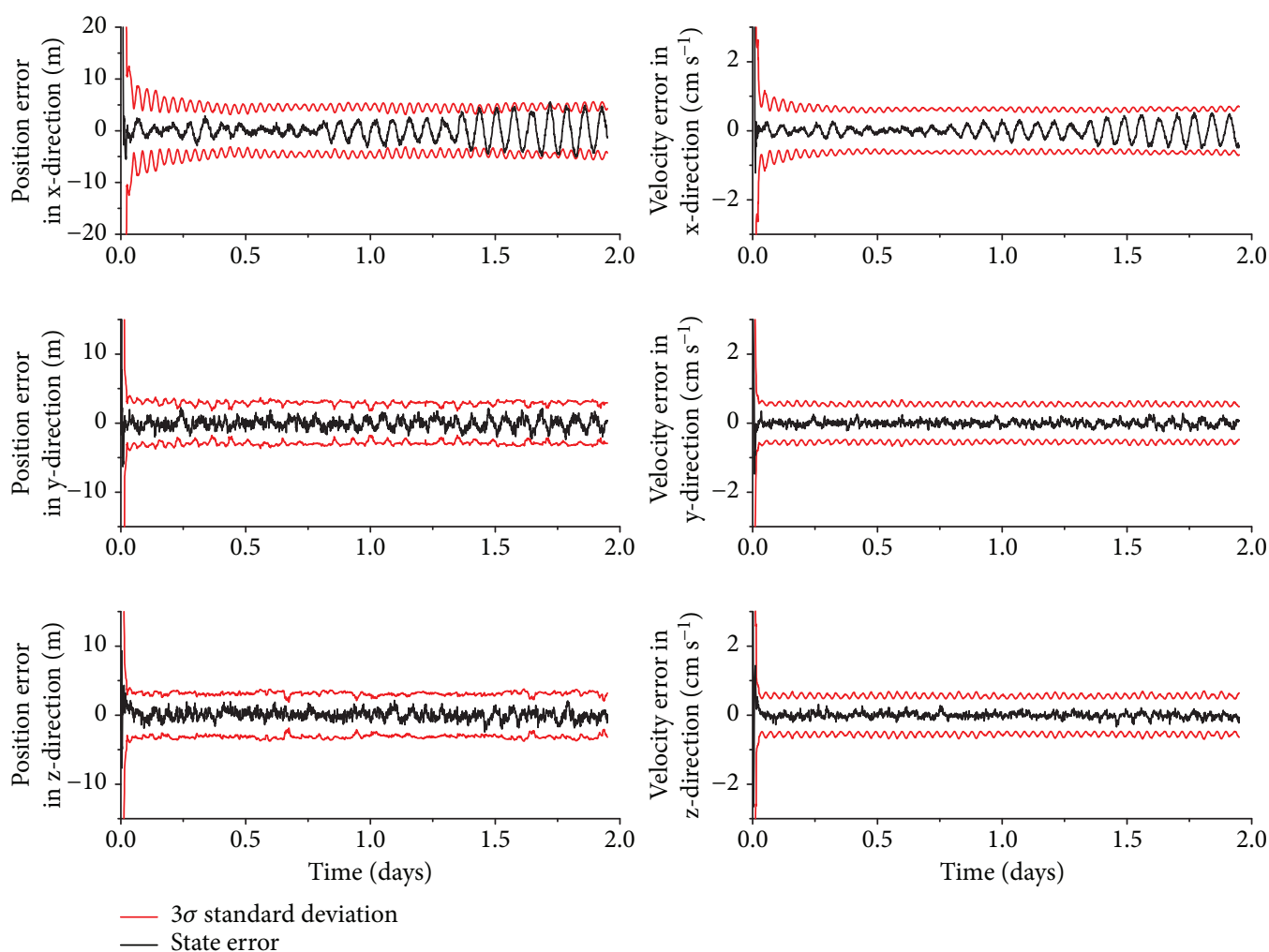

FIGURE 7: Time history of position and velocity errors of a LEO layer satellite in the J2000 coordinate frame for the case using all three observation data.
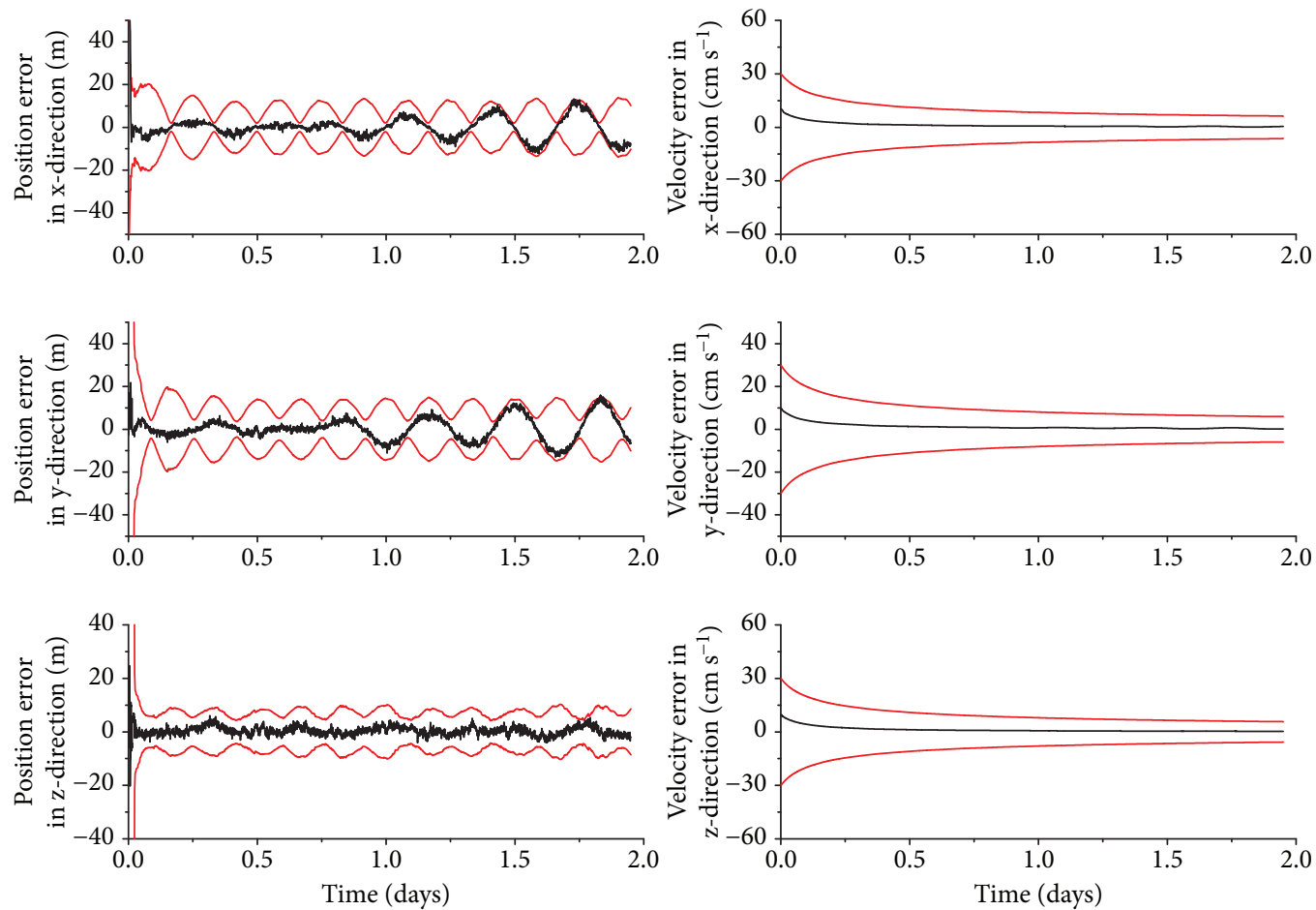

- $3 \sigma$ standard deviation

- State error

FIGURE 8: Time history of position and velocity errors of a MEO layer satellite in the J2000 coordinate frame for the case using all three observation data. 
TABLE 5: Average RMS errors of 100 Monte Carlo runs for the scenarios losing certain remote sensing observation.

\begin{tabular}{|c|c|c|c|c|c|c|}
\hline & \multicolumn{6}{|c|}{ RMS error } \\
\hline & \multicolumn{4}{|c|}{ LEO layer } & & \\
\hline & \multicolumn{2}{|c|}{$\begin{array}{l}\text { Satellites without a loss of remote } \\
\text { sensing observations }\end{array}$} & \multicolumn{2}{|c|}{$\begin{array}{c}\text { Satellites with a loss of remote } \\
\text { sensing observations }\end{array}$} & \multicolumn{2}{|c|}{ MEO layer } \\
\hline & Position (m) & Velocity $(\mathrm{cm} / \mathrm{s})$ & Position (m) & Velocity $(\mathrm{cm} / \mathrm{s})$ & Position (m) & Velocity $(\mathrm{cm} / \mathrm{s})$ \\
\hline Ideal scenario & 3.94 & 0.41 & - & - & 9.06 & 0.38 \\
\hline Scenario 1 & 9.03 & 0.94 & 9.05 & 0.94 & 25.29 & 0.66 \\
\hline Scenario 2 & 11.14 & 1.15 & 11.12 & 1.15 & 35.42 & 0.83 \\
\hline
\end{tabular}

improvement of orbit accuracy for LEO satellites. To approach real-world simulations, we investigated the orbit determination performance in situations where certain remote sensing observations are absent. Two scenarios in which only altimeter data or both imagery and altimeter data are absent on half of the LEO satellites are considered. Results show that compared to the ideal scenario, orbit determination performance of the hybrid constellation declines in both scenarios. However, for each scenario, orbit precision between LEO satellites with and without lack of measurements shows no difference. These results provide a reference for autonomous operation of constellations containing remote sensing satellites and communication satellites in future missions.

In the paper, the design of the constellation is based mainly on experience and deterministic algorithm. When more constraints are considered or more parameters need to be optimized, a heuristic algorithm could be used instead. Additionally, in order to further improve radial orbit accuracy, the altimeter crossover data could be used. How these changes would influence the orbit determination performance is worthy of in-depth analysis in future work.

\section{Appendix}

\section{A. The Derivation of (7)}

The expression of equation (7) is

$$
\left\{\begin{array}{l}
N_{d} \leq 10, \\
14 \leq \frac{N_{p}}{N_{d}} \leq 15, \\
\frac{\pi}{\arccos \left(r_{e} / a\right)} \leq N_{s} \leq \frac{2 \pi}{\arccos \left(r_{e} / a\right)}, \\
\frac{2\left(a-r_{e}\right) \tan (\mathrm{FOV} / 2)}{|\sin i|}>2 \pi r_{e} \frac{N_{d}}{\operatorname{LCM}\left(N_{d}, N_{s}\right) N_{p}}
\end{array} .\right.
$$

The first two mathematical formulations in (7) are comprehensible, which present the constraints: the revisit cycle is less than 10 days and the orbit revolution per day is more than 14 and less than 15.

For the third constraint, in order to make sure that it is visible between adjacent satellites, the distance of the Earth center to the connection line of two adjacent satellites should be larger than the Earth radius. The corresponding mathematical expression is

$$
\frac{\varphi}{2} \leq \arccos \frac{r_{e}}{a}
$$

in which $\varphi$ is the geocentric angle between two adjacent satellites. As there are $\mathrm{N}_{s}$ satellites in an orbit plane, the geocentric angle can be given as

$$
\varphi=\frac{2 \pi}{N_{s}}
$$

Substituting (A.3) into (A.2) and considering that the number of satellites required is no larger than 2 times this minimum number, the third inequality relation in (7) can be obtained:

$$
\frac{\pi}{\arccos \left(r_{e} / a\right)} \leq N_{s} \leq \frac{2 \pi}{\arccos \left(r_{e} / a\right)} .
$$

The fourth constraint is that the single-plane constellation can provide complete coverage of the Earth (except polar regions). For analyzing the coverage of satellites, the swath width $W_{\mathrm{s}}$ of a satellite needs to be known, which is

$$
W_{s}=2\left(a-r_{e}\right) \tan \frac{\mathrm{FOV}}{2} \text {. }
$$

With (A.5), the width for every satellite sweep on the equator is

$$
W_{e}=\frac{W_{\mathrm{s}}}{|\sin i|} .
$$

Since the equator is the longest latitude circle, the complete coverage of the Earth (except polar regions) is equivalent with the complete coverage of the equator region. Thus, in a revisit period, the constraint condition that satellites on an orbit plane can provide complete coverage of the Earth can be expressed as

$$
\frac{\operatorname{LCM}\left(N_{d}, N_{s}\right) N_{p}}{N_{d}} W_{e}>2 \pi r_{e},
$$

where $\operatorname{LCM}\left(N_{d}, N_{s}\right)$ represents the least common multiple of $N_{d}$ and $N_{s}$. Substituting (A.5) and (A.6) into (A.7) yields the fourth inequality relation in (7). 


\section{B. The Detailed Formula of (22)}

The detailed formula of equation $(22)$ is $\left(\left(a_{11}, \ldots, a_{33}\right)\right.$ are elements of $\left.\left(\mathbf{T}_{A} \mathbf{T}_{I}\right)^{-1}\right)$

$\mathbf{H}_{\mathbf{k}}^{\mathbf{i}}=\left[\begin{array}{cccc}-f \frac{\left(a_{31} a_{12}-a_{11} a_{32}\right)\left(r_{y}-r_{y}^{g}\right)+\left(a_{31} a_{13}-a_{11} a_{33}\right)\left(r_{z}-r_{z}^{g}\right)}{\left(a_{31}\left(r_{x}-r_{x}^{g}\right)+a_{32}\left(r_{y}-r_{y}^{g}\right)+a_{33}\left(r_{z}-r_{z}^{g}\right)\right)^{2}} & -f \frac{\left(a_{32} a_{12}-a_{12} a_{31}\right)\left(r_{x}-r_{x}^{g}\right)+\left(a_{32} a_{13}-a_{11} a_{33}\right)\left(r_{z}-r_{z}^{g}\right)}{\left(a_{31}\left(r_{x}-r_{x}^{g}\right)+a_{32}\left(r_{y}-r_{y}^{g}\right)+a_{33}\left(r_{z}-r_{z}^{g}\right)\right)^{2}} & -f \frac{\left(a_{33} a_{11}-a_{13} a_{31}\right)\left(r_{x}-r_{x}^{g}\right)+\left(a_{32} a_{13}-a_{12} a_{33}\right)\left(r_{y}-r_{y}^{g}\right)}{\left(a_{31}\left(r_{x}-r_{x}^{g}\right)+a_{32}\left(r_{y}-r_{y}^{g}\right)+a_{33}\left(r_{z}-r_{z}^{g}\right)\right)^{2}} & (\mathbf{0})_{1 \times 3} \\ -f \frac{\left(a_{31} a_{22}-a_{21} a_{32}\right)\left(r_{y}-r_{y}^{g}\right)+\left(a_{31} a_{23}-a_{21} a_{33}\right)\left(r_{z}-r_{z}^{g}\right)}{\left(a_{31}\left(r_{x}-r_{x}^{g}\right)+a_{32}\left(r_{y}-r_{y}^{g}\right)+a_{33}\left(r_{z}-r_{z}^{g}\right)\right)^{2}} & -f \frac{\left(a_{32} a_{22}-a_{22} a_{31}\right)\left(r_{x}-r_{x}^{g}\right)+\left(a_{32} a_{23}-a_{21} a_{33}\right)\left(r_{z}-r_{z}^{g}\right)}{\left(a_{31}\left(r_{x}-r_{x}^{g}\right)+a_{32}\left(r_{y}-r_{y}^{g}\right)+a_{33}\left(r_{z}-r_{z}^{g}\right)\right)^{2}} & -f \frac{\left(a_{33} a_{21}-a_{23} a_{31}\right)\left(r_{x}-r_{x}^{g}\right)+\left(a_{32} a_{23}-a_{22} a_{33}\right)\left(r_{y}-r_{y}^{g}\right)}{\left(a_{31}\left(r_{x}-r_{x}^{g}\right)+a_{32}\left(r_{y}-r_{y}^{g}\right)+a_{33}\left(r_{z}-r_{z}^{g}\right)\right)^{2}} & (\mathbf{0})_{1 \times 3}\end{array}\right]$.

\section{Data Availability}

The data used to support the findings of this study are available from the corresponding author upon request.

\section{Conflicts of Interest}

The authors declare that there is no conflict of interest regarding the publication of this paper.

\section{Acknowledgments}

This work was carried out with financial support from the National Basic Research Program 973 of China (2015CB857100), the Base of National Defence Scientific Research Fund (Nos. 2016110C019 and JCKY2016203C067), and the National Natural Science Foundation (No. 11603011).

\section{References}

[1] A. L. Jennings and H. Diniz, "Global navigation satellite system design exploration using a multi-objective genetic algorithm," in AIAA SPACE 2015 Conference and Exposition, p. 4622, Pasadena, CA, USA, 2015.

[2] H. Xu, X. Zhang, and X. Zhan, "Analyzing RAIM performance for an MEO/GEO hybrid GNSS constellation," in IET International Communication Conference on Wireless Mobile and Computing (CCWMC 2011), pp. 465-467, Shanghai, China, November 2011.

[3] S. Chan, A. T. Samuels, N. B. Shah, J. E. Underwood, and O. L. De Weck, "Optimization of hybrid satellite constellations using multiple layers and mixed circular-elliptical orbits," in 22nd AIAA International Communications Satellite Systems Conference \& Exhibit 2004, pp. 9-12, Monterey, CA, USA, May 2004.

[4] M. Luglio and W. Pietroni, "Optimization of double-link transmission in case of hybrid orbit satellite constellations," Journal of Spacecraft and Rockets, vol. 39, no. 5, pp. 761-770, 2002.

[5] E. Fahnestock and R. S. Erwin, "Optimization of hybrid satellite and constellation design for GEO-belt space situational awareness using genetic algorithms," in Proceedings of the 2005, American Control Conference, 2005, pp. 2110-2115, Portland, OR, USA, June 2005.
[6] R. L. White, M. B. Adams, E. G. Geisler, and F. D. Grant, "Attitude and oribit estimation using stars and landmarks," IEEE Transactions on Aerospace and Electronic Systems, vol. AES11, no. 2, pp. 195-203, 1975.

[7] M. Straub and J. Christian, "Autonomous optical navigation for Earth-observing satellites using coastline matching," in AIAA Guidance, Navigation, and Control Conference, Kissimmee, FL, USA, 2015.

[8] M. Li, B. Xu, and L. Zhang, "Orbit determination for remotesensing satellites using only optical imagery," International Journal of Remote Sensing, vol. 38, no. 5, pp. 1350-1364, 2017.

[9] M. Li and B. Xu, "Autonomous orbit and attitude determination for Earth satellites using images of regular-shaped ground objects," Aerospace Science and Technology, vol. 80, pp. 192202, 2018.

[10] G. H. Born, B. D. Tapley, and M. L. Santee, "Orbit determination using dual crossing arc altimetry," Acta Astronautica, vol. 13, no. 4, pp. 157-163, 1986.

[11] F. G. Lemoine, D. D. Rowlands, S. B. Luthcke et al., "Precise orbit determination for Geosat follow-on using satellite laser ranging data and intermission altimeter crossovers," in 2001 Flight Mechanics Symposium, Greenbelt, MD, USA, 2001.

[12] F. Markley and U. Naval, "Autonomous navigation using landmark and intersatellite data," in AIAA/AAS Astrodynamics Conference, Seattle, WA, USA, 1984.

[13] M. L. Psiaki, "Autonomous orbit determination for two spacecraft from relative position measurements," Journal of Guidance, Control, and Dynamics, vol. 22, no. 2, pp. 305-312, 1999.

[14] R. Li, H. Qiu, and K. Xiong, "Autonomous navigation for constellation based on inter-satellite ranging and directions," in IECON 2017 - 43rd Annual Conference of the IEEE Industrial Electronics Society, pp. 2985-2990, Beijing, China, November 2017.

[15] X. Kai, W. Chunling, and L. Liangdong, “Autonomous navigation for a group of satellites with star sensors and inter-satellite links," Acta Astronautica, vol. 86, pp. 10-23, 2013.

[16] X. Kai, W. Chunling, and L. Liangdong, "The use of X-ray pulsars for aiding navigation of satellites in constellations," Acta Astronautica, vol. 64, no. 4, pp. 427-436, 2009.

[17] S. Wang and P. Cui, "Autonomous orbit determination using pulsars and inter-satellite ranging for Mars orbiters," in 2018 IEEE Aerospace Conference, Big Sky, MT, USA, March 2018.

[18] M. Capderou, Handbook of Satellite Orbits: From Kepler to GPS, Springer Science \& Business, 2014. 
[19] https://directory.eoportal.org/web/eoportal/satellite-missions/ $\mathrm{d} / \mathrm{dmc}-3$.

[20] D. Mortari, M. P. Wilkins, and C. Bruccoleri, "The Flower Constellations," Journal of Astronautical Sciences, vol. 52, pp. 107-127, 2004.

[21] M. P. Wilkins and D. Mortari, "Flower Constellation set theory part ii: secondary paths and equivalency," IEEE Transactions on Aerospace and Electronic Systems, vol. 44, no. 3, pp. 964976, 2008.

[22] J. G. Walker, Continuous Whole-Earth Coverage by CircularOrbit Satellite Patterns, Royal Aircraft Establishment Farnborough (United Kingdom), 1977.

[23] B. Schutz, B. Tapley, and G. H. Born, Statistical Orbit Determination, Academic Press, 2004.

[24] http://calvalportal.ceos.org/calibration-test-sites. 


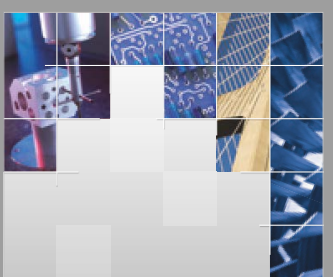

\section{Enfincering}
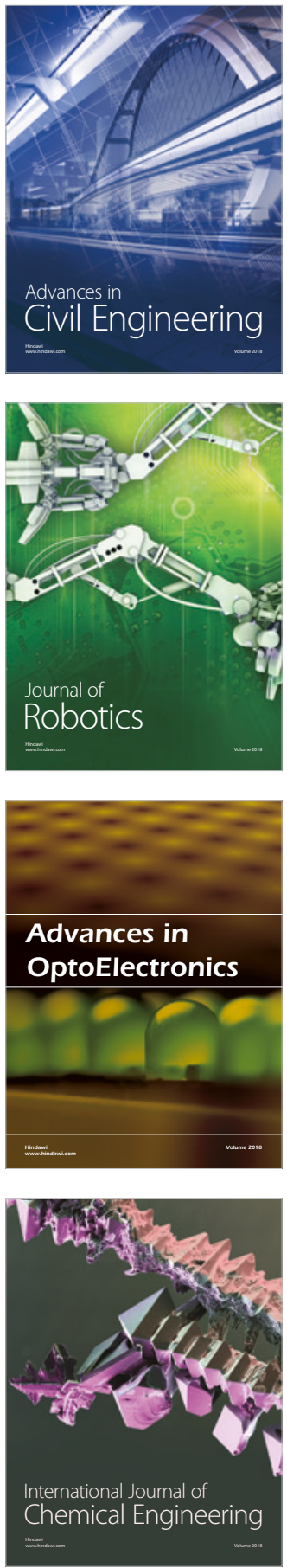

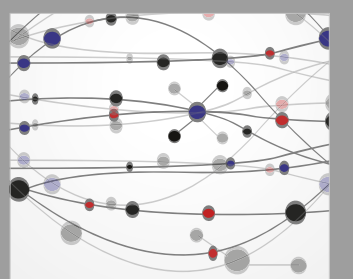

\section{Rotating \\ Machinery}

The Scientific World Journal

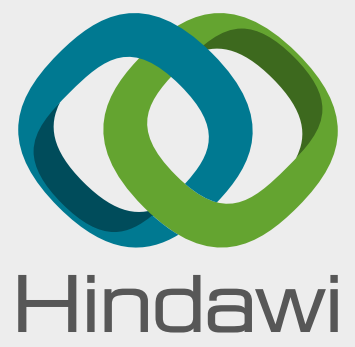

Submit your manuscripts at

www.hindawi.com
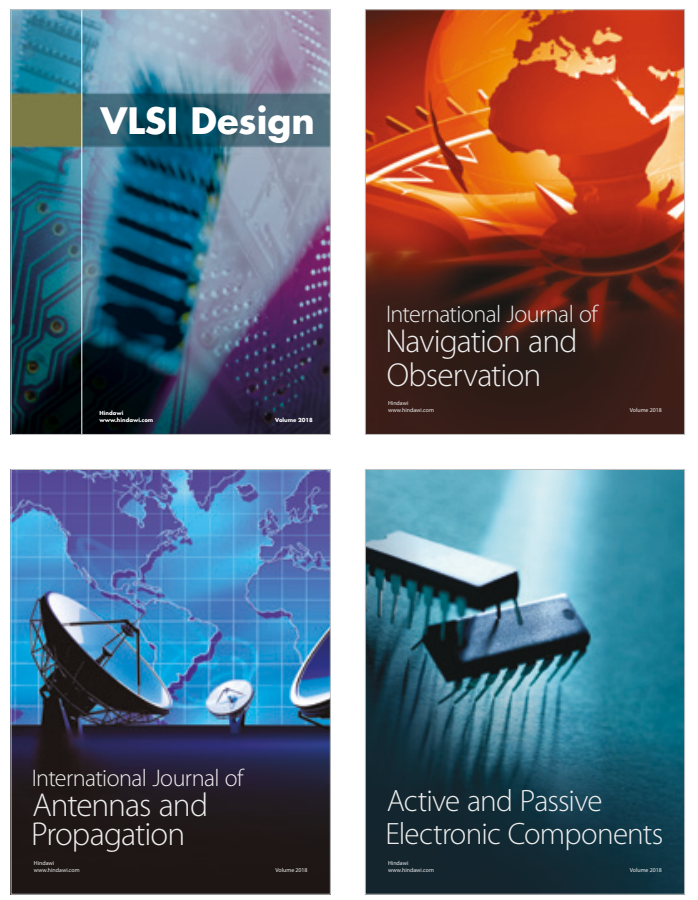
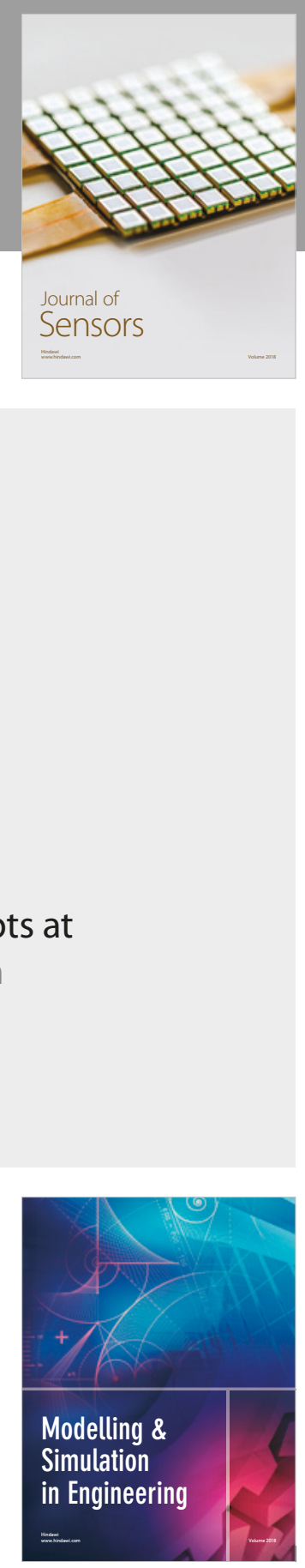

\section{Advances \\ Multimedia}
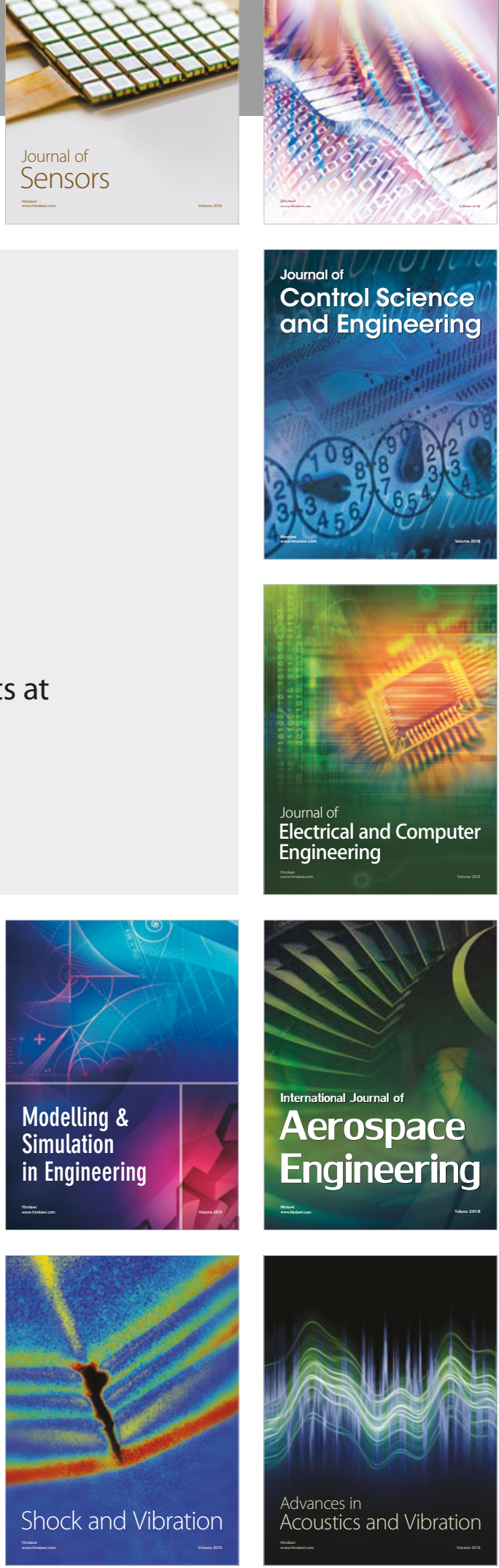